\title{
Zwitter, Buckliger und Grimassenmensch - abweichende Körperlichkeit bei Johann Wolfgang von Goethe und Victor Hugo (Wilhelm Meisters Lehrjahre, Notre-Dame de Paris, L'Homme qui rit)
}

L'hermaphrodite, le bossu, l'homme grimaçant - la déviance physique chez Johann Wolfgang von Goethe et Victor Hugo (Wilhelm Meisters Lehrjahre, Notre-Dame de Paris, L'Homme qui rit) Hybrid, Humpback and Gurner - Divergent Physicality in Johann Wolfgang von Goethe and Victor Hugo (Wilhelm Meisters Lehrjahre, NotreDame de Paris, L'Homme qui rit)

Hans Richard Brittnacher

\section{OpenEdition \\ Journals}

Édition électronique

URL : https://journals.openedition.org/ceg/10272

DOI : $10.4000 /$ ceg. 10272

ISSN : 2605-8359

Éditeur

Presses Universitaires de Provence

Édition imprimée

Date de publication : 20 janvier 2020

Pagination : 221-236

ISBN : 979-10-320-0253-7

ISSN : 0751-4239

\section{Référence électronique}

Hans Richard Brittnacher, „Zwitter, Buckliger und Grimassenmensch - abweichende Körperlichkeit bei Johann Wolfgang von Goethe und Victor Hugo (Wilhelm Meisters Lehrjahre, Notre-Dame de Paris, L'Homme qui rit)", Cahiers d'Études Germaniques [Online], 78 | 2020, Online erschienen am: 21 Oktober 2021, abgerufen am 12 Februar 2022. URL: http://journals.openedition.org/ceg/10272 ; DOI: https:// doi.org/10.4000/ceg.10272 


\section{Zwitter, Buckliger und Grimassenmensch - abweichende Körperlichkeit bei Johann Wolfgang von Goethe und Victor Hugo (Wilhelm Meisters Lehrjahre, Notre-Dame de Paris, L'Homme qui rit)}

Hans Richard BRITTNACHER

Freie Universität Berlin

\section{Das Klassische und das Romantische, oder: das Gesunde und das Kranke?}

Goethes Aversion gegen Krankheit, erst recht gegen deformierte Körperlichkeit ist bekannt. Im Gespräch mit Eckermann begründete er seine Abneigung gegen die Romantik mit der vermuteten Nähe ihrer Poetik zum Pathologischen: „Das Klassische nenne ich das Gesunde, und das Romantische das Kranke." ${ }^{1}$ Polemisch fällt Goethes Urteil über die romantischen Dichter aus: „Die [romantischen] Poeten schreiben alle, als wären sie krank und die ganze Welt ein Lazarett. [...] Ich habe ein gutes Wort gefunden [...] um diese Herren zu ärgern. Ich will ihre Poesie die Lazarett-Poesie nennen." 2 Nicht bezeugt, nur von Ernst Wilhelm Webers sonst sehr zuverlässiger Geschichte des Weimarischen Theaters überliefert ist der Gefühlsausbruch, mit dem Goethe auf Kleists Käthchen von Heilbronn reagierte. Goethe hatte Johannes Falk gebeten, als eine Art literarischer Vorkoster zu agieren und ihm seinen Eindruck mitzuteilen. Erst danach „will ich einmal mit mir zurate gehen, ob ich es denn auch lesen kann. Beim Lesen seiner ,Penthesilea“ bin ich neulich gar zu übel weggekommen."3 Eine Amazonin, die im Liebesrausch

1. Johann Peter Eckermann, Gespräche mit Goethe in den letzten Jahren seines Lebens, hrsg. von Ernst Beutler, Gedenkausgabe der Werke, Briefe und Gespräche (GA), Bd. 24, Zürich, Artemis-Verlag, 1976 [1948], 2. April 1829, S. 332. Grundsätzlich zum Thema: Erich Jenisch, „,Das Klassische nenne ich das Gesunde, und das Romantische das Kranke؛ Goethes Kritik der Romantik“, in Goethe Jahrbuch 19, 1957, S. 50-79. Vgl. zum Thema auch: Hans-Dietrich Dahnke, „Gesundheit/Krankheit“, in Bernd Witte, Theo Buck, Hans-Dietrich Dahnke u.a. (Hrsg.), Goethe Handbuch 4/1: Personen, Sachen, Begriffe, Stuttgart, Metzler, 1998, S. 283-385.

2. Eckermann, Gespräche, 24. September 1827, S. 268.

3. Johann Wolfgang von Goethe, Weimarer Ausgabe (WA), Bd. I, 21, S. 332. 
den geliebten Achill in Stücke reißt und verschlingt, konnte schwerlich Goethes Gefallen finden, auch wenn Kleist ihm sein Drama bekanntlich „auf den Knieen seines Herzens" ${ }^{4}$ offeriert hatte. Aber auch das bedingungslos den Ritter Wetter vom Strahl liebende, schlafwandelnde Käthchen konnte Goethes Abscheu nicht mildern. Nach der Lektüre weniger Szenen in einem geliehenen Exemplar war es um seine Contenance geschehen. Mit den Worten „Ein wunderbares Gemisch von Sinn und Unsinn! Die verfluchte Unnatur!“ warf er das Buch ins Kaminfeuer.

An der Verlässlichkeit der Anekdote sind erhebliche Zweifel angemeldet worden: schwer vorstellbar sei, dass Goethe geborgte Bücher ins Feuer geworfen habe, etc. Hier aber sollte eine italienische Redensart ihr Recht erhalten: se non è vero è ben trovato - mag es auch nicht wahr sein, so ist es doch gut erfunden. Tatsächlich trifft die Anekdote den Nagel auf den Kopf. Sie zeigt Goethes geradezu entschlossene Wahrnehmung der Literatur Kleists als eines monströsen Angriffs auf Dezenz und Beherrschung, und die seiner Figuren und ihres Autors als pathologische, zu Schwärmerei, Hypochondrie oder Schwermut neigende Grenzgänger - ihnen gebührt kein Platz in dem, was Goethe und Schiller als literarischen Standard in Weimar etabliert hatten. ${ }^{5}$ In diesem Sinne äußerte er sich auch noch 1826, fünfzehn Jahre nach Kleists Tod, anlässlich einer von Ludwig Tieck besorgten Ausgabe der Werke Kleists: „Mir erregte dieser Dichter, bei dem reinsten Vorsatz einer aufrichtigen Teilnahme, immer Schauder und Abscheu, wie ein von der Natur schön intentionierter Körper, der von einer unheilbaren Krankheit ergriffen wäre.“

\section{6: Mignon (Johann Wolfgang von Goethe, Wilhelm Meisters Lehrjahre)}

Damit hat Goethe, bezogen auf Kleist, Kategorien des Pathologischen namhaft gemacht, die durchaus auch eine Gestalt in seinem eigenen Werk treffen - sogar eine besonders wichtige Gestalt in einem besonders programmatischen Roman, nämlich Mignon in Wilhelm Meisters Lehrjahren. Verärgert über eine Bemerkung der Madame de Staël, die von Mignon als einer „épisode charmant“ gesprochen hatte, behauptete Goethe gegenüber dem Kanzler Müller, nur dieses Charakters wegen den ganzen Roman geschrieben zu haben. ${ }^{6}$ Der Roman aber

4. Kleist an Goethe, 24. Januar 1808; vgl. Heinrich von Kleist, Sämtliche Werke und Briefe, Bd. 2: Dramen 1808-1811, Frankfurter Ausgabe, hrsg. von Ilse-Marie Barth, Klaus Müller-Salget, Walter Müller-Seidel und Hinrich C. Seeba, Frankfurt a. M., Deutscher Klassiker Verlag, 1987, S. 694.

5. Zum Selbstverständnis der Weimarer Dioskuren als einer „ecclesia militans“ gegen den schlechten Geschmack der Zeit vgl. Terence James Reed: „Ecclesia militans: Weimarer Klassik als Opposition“, in Winfried Barner, Eberhard Lämmert, Norbert Oellers (Hrsg.), Unser Commercium. Goethes und Schillers Literaturpolitik, Stuttgart, Cotta, 1984, S. 37-53; exemplarisch zu Schiller: Hans Richard Brittnacher, „Die Austreibung des Populären - Schillers Bürger-Kritik“, in Goethe Yearbook 15, 2018, S. 97-107.

6. Kanzler Friedrich von Müller, Unterhaltungen mit Goethe, hrsg. von Renate Grumach, München, C.H. Beck, 1995, S. 16. 
muss diese so wichtige Figur zuletzt doch sterben lassen, weil die Welt, die er schildert, für Gestalten wie sie keinen Lebensraum vorsieht. ${ }^{7}$ Ihr Schicksal, das Wilhelm Meisters Lehrjahre (1796) mitteilt, liefert ein - vom Autor wohl kaum intendiertes - Beispiel für die Erfahrung und Zähmung des Monströsen und damit einen Einblick in die ästhetische und soziale Praxis der Sattelzeit bei der Thematisierung pathologischer Körperlichkeit. In seinen Notizheften hatte Goethe zu der kleinen, knabenhaften Mignon, eine der farbigsten Figuren in seinem Erzählwerk, eine die Irritation dieser Figur charakterisierende Bestimmung festgehalten: „Wahnsinn des Missverhältnisses.“ ${ }^{8}$ Zwar gilt Wilhelm Meisters Lehrjahre als Prototyp des Bildungsromans, eines Romantyps von erheblichen integrativen Kompetenzen, aber an Mignon ist alles so dissonant und diskrepant, dass sie in der zwar geistvollen, aber doch blutleeren Welt der Moderne, in ihrem maßvollen Leben und ihrer geregelten Geselligkeit, keinen dauerhaften Platz finden kann: Das „Rätsel“9 ${ }^{9}$ wie Mignon von anderen genannt wird, sogar von denen, die selbst einen so unkonventionellen Lebensstil pflegen wie die leichtsinnige Philine, hat nicht einmal einen richtigen Namen - sie ist, was sie genannt wird: „Mignon“, d.h. klein, eine Liebhaberei, die Zuneigung mit Gefälligkeiten entgilt. ${ }^{10}$ Wilhelm ist bezaubert von ihr, seine

Augen und sein Herz wurden unwiderstehlich von dem geheimnisvollen Zustande dieses Wesens angezogen. Er schätzte sie zwölf bis dreizehn Jahre; ihr Körper war gut gebaut, nur daß ihre Glieder einen stärkern Wuchs versprachen oder einen zurückgehaltenen ankündigten. ${ }^{11}$

Verwirrender noch als die Mischung aus Liebreiz und dunklem Zauber ist die sexuelle Uneindeutigkeit Mignons: Wilhelm „sah die Gestalt mit Verwunderung an und konnte nicht mit sich einig werden, ob er sie für einen Knaben oder für ein Mädchen erklären sollte.“" ${ }^{12}$ Die irritierende geschlechtliche Neutralität Mignons wird noch verstärkt durch die Neigung, nur Knabenkleidung zu tragen und wie Jungen zu springen und zu klettern. Aber den Theaterschauspielern um Melina, die sich für etwas Besseres als die Gaukler halten, und erst recht dem kaltherzigen Spötter Jarno erscheint Mignon als Zigeunerin und als „albernes, zwitterhaftes Geschöpf“"13.

7. Vgl. dazu grundsätzlich Hans Richard Brittnacher, „Mythos und Devianz in Wilhelm Meisters Lehrjahre“, Leviathan 1, 1986, Sonderheft „Goethe“, S. 96-110.

8. Goethes Werke, WA, Bd. I, 21, S. 332.

9. Ich zitiere Goethes Roman mit der vorangestellten Sigle WML nach der Ausgabe: Goethes Werke, Hamburger Ausgabe (HA), Bd. 7, hrsg. von Erich Trunz, München, C.H. Beck, 1973. Hier WML 98.

10. Als mignons wurden die Liebhaber der französischen Könige bezeichnet - im Gegensatz zum protégé ist beim mignon auch die Idee sexueller Dienstleistungen präsent. Vgl. Nicolas Le Roux, La Faveur du roi. Mignons et courtisans au temps des derniers Valois (vers 1547-vers 1589), Seyssel, Champ Vallon, 2001. Im Weimarer gossip wurde auch der von vielen beneidete und beargwöhnte Goethe gelegentlich als Mignon des Herzogs von Weimar bezeichnet.

11. WML 98.

12. WML 91.

13. WML 193. 
Mignon steht zwischen den Geschlechtern und zwischen den Generationen, eine ,junge, schwarzköpfige, düstere Gestalt" ${ }^{14}$. Inmitten der entzauberten Welt der Moderne, deren Überlegenheit im Roman außer Zweifel steht, erhält mit Mignon noch einmal das Geheimnis einen letzten Auftritt. Mignon ist nicht nur geschlechtlich unentschieden, sie steht auch zwischen einer südlichen Kunstwelt und einer nördlichen Mythologie: ein Wesen von rastloser Beweglichkeit, zuweilen rasend-tänzerisch wie eine Mänade, und dann doch wieder von so zerbrechlicher Physis, dass, sie, kaum bekannt geworden mit dem Aufkeimen der Liebe, jung stirbt. Lange Jahre hat sie, das Produkt eines Geschwisterinzests und von Fahrenden entführtes Kind, als Artistin in einer Seiltänzergruppe gelebt. Als die Truppe in der Stadt gastiert, in der Wilhelm sich mit seinen Schauspielern aufhält, wird er Zeuge der brutalen Dressur des Artistenkindes:

Mit Entsetzen erblickte er, als er sich durchs Volk drängte, den Herrn der Seiltänzergesellschaft, der das interessante Kind bei den Haaren aus dem Haus zu schleppen bemüht war und mit seinem Peitschenstiel unbarmherzig auf den kleinen Körper losschlug. ${ }^{15}$

Durch sein beherztes Dazwischentreten kann Wilhelm die Züchtigung unterbinden und kauft dem fahrenden Mann kurzerhand das sonderbare, orientalisch kostümierte Kind ab. $\mathrm{Zu}$ ihrem neuen Herrn fasst die wie eine Sklavin freigekaufte Mignon ein inniges Verhältnis, sie trägt von nun an ihre Kleidung - vorzugsweise Knabenkleidung - nur noch in seinen Farben, sie schläft an der Schwelle zu seiner Kammer und bittet ihn inständig, sie nie zu verlassen. Ihr oft gehemmtes und verschlossenes Wesen entlädt sich mitunter in fast ekstatischen Gefühlsausbrüchen und einem Strom von Tränen. Als eine besondere Ehre darf Wilhelm es empfinden, dass sie ihm den Eiertanz vorführt, dessen Aufführung sie ihrem Patron verweigert hat. Es ist eine seltsame Darbietung, die wenig tänzerische Anmut, aber viel befremdliche Archaik zeigt. Zu den Klängen eines Fandangos und zum Schlagen der Kastagnetten tanzt sie mit verbundenen Augen zwischen den auf dem Boden ausgelegten Eiern:

Behende, leicht, rasch, genau führte sie den Tanz. Sie trat so scharf und so sicher zwischen die Eier hinein, bei den Eiern nieder, daß man jeden Augenblick dachte, sie müsse eins zertreten. [...] Streng, scharf, trocken, heftig und in sanften Stellungen mehr feierlich als angenehm zeigte sie sich. ${ }^{16}$

Obwohl es Wilhelm schwerfällt, sich von dem anhänglichen Wesen zu trennen, gibt er das herzkranke Mädchen in bester erzieherischer Absicht an die Mitglieder der Sozietät von Turm, die sich mit großem Eifer, aber wenig Erfolg des widerspenstigen Kindes annehmen. Als großer therapeutischer Fortschritt erscheint ihnen, dass Mignon die Kleidung gewechselt hat: „,Sie geht nunmehr in Frauenkleidern, vor denen sie sonst einen so großen Abscheu zu

\footnotetext{
14. WML 92.

15. WML 103.

16. WML $115 f$.
} 
haben schien.“ 17 Was der Erfolg einer Erziehung in heteronormativer Absicht $\mathrm{zu}$ bestätigen scheint, zeigt freilich einen ironischen Gegensinn: Mignon, das Zwitterwesen, das weder Junge noch Mädchen ist, scheidet schließlich in einer Gestalt aus dem Leben, die als einzige zu Goethes Zeit eine wohl akzeptable Form des Zwittertums verkörpert, als Engel. Ihr berühmtes Lied „So laßt mich scheinen, bis ich werde“"18, das sie in einer Art Sonntagsschule, die Natalie mit den Bauernkindern der Umgebung abhält, im Engelskostüm mit Flügeln an den Schultern, singt, hat für die befremdliche Andersheit dieser Figur zu guter Letzt ein versöhnliches Bild gefunden: Friedrich Wilhelm von Schadow hat es gemalt - an die Stelle des Hermaphroditismus eines bunten Zirkuskindes in seidenem Westchen und buntem Flitter ist die ätherische Erscheinung eines Engels getreten, der schon nicht mehr von dieser Welt ist.

Damit ist die Prozedur der Normalisierung eines „zwitterhaften Geschöpfes“ noch nicht abgeschlossen. Nachdem sie gestorben ist, wird sie, deren charakteristisches Merkmal doch die Unverfügbarkeit war - verfügbar wollte sie nur dem einen sein, der sie nicht wollte - schließlich allen dauerhaft verfügbar. In einem erzählerischen Kunstgriff von grotesker Befremdlichkeit entschließt sich der Erzähler, ihre sterbliche Hülle einbalsamieren zu lassen: „Eine balsamische Masse ist durch alle Adern gedrungen und färbt nun an der Stelle des Bluts die so früh verblichenen Wangen.“ " 19 Durchgeführt wird die Prozedur vom Abbé, einem vom Segen des Fortschritts überzeugten Aufklärer, dem die zu Tode therapierte Mignon gerade gelegen kommt, dem Rätsel des Lebens einen Platz in der Totenkammer der Anomalien zuzuweisen. Das mumifizierte Schaustück, endlich und unwiderruflich verfügbar, präsentiert er den Anwesenden, ihrem Beschützer Wilhelm, ihrer Erzieherin Nathalie und dem Marchesen, ihrem leiblichen Oheim, mit Worten, die gewiss nicht zufällig an einen Zirkusdirektor erinnern, der seine nächste Attraktion anpreist: „Treten Sie näher, meine Freunde, und sehen Sie das Wunder der Kunst und Sorgfalt!“20

Der Abbé überführt die Irritation angesichts der unverfügbaren Fremdheit von Mignons Körper, ihr Geheimnis, das sich als Störung, Widersetzlichkeit und Desorientierung zur Geltung brachte, in die gelassene Anschauung eines toten, dauerhaft der Betrachtung preisgegebenen Objekts. Das geheimnisvolle Geschöpf erhält so als Erinnerung an den früheren Status der Poesie einen Ort des Gedenkens, was monströs und befremdlich an ihr war, ist nun in einem Schausaal als Präparat konserviert. Das Interesse an der im Saal der Vergangenheit aufgebahrten Mignon ermöglicht in ästhetischer Hinsicht Erinnerung, in teratologischer und wissensgeschichtlicher Perspektive Anschauung für einen Wechsel von Deutungsmustern. Mignon steht an der Schwelle des Übergangs von der Präformationstheorie, die im Monstrum ein einmaliges Exemplar sah, Ausdruck eines unerforschlichen göttlichen Willens und der stupenden Vielfalt der

17. WML 514.

18. WML $515 f$.

19. WML 577.

20. Ibid. 
Schöpfung, zu einer epigenetischen Theorie, die aus dem Studium des Präparats Rückschlüsse über die Entwicklung natürlicher Organismen zu gewinnen hofft. ${ }^{21}$

\section{1: Quasimodo (Victor Hugo, Notre-Dame de Paris)}

Als Reminiszenz an die Figur der Mignon lässt sich die kleine Esmeralda in Victor Hugos Roman Notre-Dame de Paris lesen, ${ }^{22}$ einem Werk, das dennoch Goethes Zustimmung deutlich verfehlte. Hugo hat im Vorwort seines Dramas Cromwell (1827) sein poetologisches Programm erläutert, mit den rigiden Stilprinzipien der französischen Klassik zu brechen und seinen Anspruch auf eine extravagante Ästhetik artikuliert, die das Sublime und das Groteske zu vereinen hat - diesem Programm folgt auch der Roman, sowohl mit seinem Schauplatz, der architektonisch uneinheitlichen und doch überwältigenden Kathedrale NotreDame, wie mit seinem Protagonisten, dem zyklopischen Glöckner, wie auch mit der turbulenten Handlung. ${ }^{23}$ Die Ästhetik Hugos mit ihrer Rehabilitation der Schauwerte des Hässlichen und der exzessiven Fabuliersucht des Autors hat Goethes entschlossene Missbilligung herausgefordert, die er seinem Eckermann zu Protokoll gab:

Ich habe in diesen Tagen seine Notre-Dame de Paris gelesen und nicht geringe Geduld gebraucht, um die Qualen auszustehen, die diese Lektüre mir gemacht hat. Es ist das abscheulichste Buch, das je geschrieben worden! [...] ohne alle Natur und alle Wahrheit. Seine vorgeführten sogenannten handelnden Personen sind keine Menschen mit lebendigem Fleisch und Blut, sondern elende hölzerne Puppen, mit denen er umspringt, wie er Belieben hat, und die er allerhand Verzerrungen und Fratzen machen lässt, so wie er es für seine beabsichtigten Effekte eben braucht. ${ }^{24}$

Die Empörung Goethes - der freilich das Urteil Lamartines entgegenzuhalten wäre, der Hugo als den „Shakespeare des Romans“ bezeichnete - mag auch dem Umstand geschuldet sein, dass Hugo in Notre-Dame gewissermaßen eine abenteuergesättigte Variante seiner Mignongeschichte erzählt. Denn wie Goethes Mignon auf Cervantes' La Gitanilla von 1613 zurückgeht, ist auch in der liebreizenden Esmeralda aus Hugos Notre-Dame eine Verkörperung und Weitererzählung der Preziosa zu erkennen, auch sie wie Goethes Mignon und Cervantes' Preziosa ein „unglückliche[s] Geschöpf ohne Vaterland, ohne Familie, ohne Heimat.“ 25

21. Vgl. zu diesem Paradigmenwechsel Michael Hagner, „Monstrositäten haben eine Geschichte“, in Hagner (Hrsg.), Der falsche Körper. Beträge zu einer Geschichte der Monstrositäten, Göttingen, Wallstein, 1992, S. 7-20, hier v.a. S. 15.

22. Vgl. detaillierter zu den Parallelen beider Romane Hans Richard Brittnacher, „Todgeweiht und sterblich - Mignon-Reminiszenzen in der französischen Literatur“, in Goethe Jahrbuch 134, 2017, S. 187-198.

23. Victor Hugo, La Préface de Cromwell, hrsg. von Maurice Souriau, Genf, Slatkine reprints, 1973.

24. Eckermann, Gespräche, 27. Juni 1831, S. 760.

25. Ich zitiere nach der deutschen Ausgabe: Victor Hugo, Der Glöckner von Notre-Dame, Zürich, Diogenes, 1983, S. 382/ „malheureuse créature, sans patrie, sans familie, sans foyer“ (Victor Hugo, Notre-Dame de Paris. 1482. Les Travailleurs de la mer, textes établis, présentés et annotés 
Das blutjunge Zigeunermädchen ${ }^{26}$ erobert im spätmittelalterlichen Paris mit seinem Tanz die Herzen der Zuschauer - wie Mignon eben auch. Während diese jedoch in ihrem Trotz und ihrer seelischen Verschlossenheit die Kunst für sich bewahren will, bietet Esmeralda sie - sehr viel stärker als Mignon dem Klischee der ostentativ mit ihren weiblichen Reizen kokettierenden Zigeunerin entsprechend - dem Publikum gerne an. Dennoch sind die Details des Tanzes in ihrer Parallelität schwer zu übersehen: Auch Esmeralda rollt vor ihrer Darbietung einen Teppich aus, auch ihr Tanz wird mit einem Schwertertanz verglichen, auch er lässt sich vom Fandango den Takt vorgeben, und er hat eine betörende, nachgerade verhexende Wirkung. Esmeraldas Tanz ist wie der Mignons geeignet, das verstockte Innere der männlichen Zuschauer in Bewegung zu setzen, den Dichter Pierre Gringoire weinen zu lassen, so wie Wilhelm gelegentlich von Mignons Liedern zu Tränen gerührt ist. Aber während Mignons Fremdartigkeit auch durch die Bravour des Tanzes nicht beeinträchtigt wird, erweist sich die hinreißend tanzende Esmeralda als erotischer Blickfang: „Ob dieses junge Mädchen ein menschliches Wesen, oder eine Fee, oder ein Engel war, das konnte Gringoire [...] auf den ersten Blick nicht feststellen, derart bezaubert war er durch diese blendende Erscheinung." ${ }^{27}$ Die erotische Faszination, die Esmeralda auf nur wenigen Seiten auf drei grundverschiedene Männer ausübt, auf den eitlen Phœbus, den dämonischen Frollo und den grobschlächtigen Quasimodo, hat Hugo in einer Kapitelüberschrift zum Ausdruck gebracht, die sich freilich auch als ironischer Hinweis auf die Einfältigkeit des männlichen Begehrens deuten lässt: „Drei verschieden geartete Menschenherzen.“28

Auch Esmeraldas Äußerungen, ein Kauderwelsch aus unterschiedlichen Dialekten und Sprachen und die eigentümliche Ironie, mit der sie Fragen zu beantworten pflegt, erinnern an Mignon Spiel mit der Sprache des Rätsels, so wie ihre Frömmigkeit an Mignons Verehrung der Muttergottes. Wie Mignon in ihrer Fremdartigkeit Objekt von Anfeindungen und Misstrauen wird, muss auch Esmeralda ertragen, als Zigeunerin diskriminiert zu werden: und wie Mignon sich

par Jacques Seebacher et Yves Cohin, Paris, Gallimard, 1975). Ich zitiere nach dieser Ausgabe unter Verwendung der Sigle NDdP. Hier NDdP 365.

26. Die Angehörigen der Sinti und Roma verbitten sich mit gutem Grund die Verwendung des diskriminierenden Terminus „Zigeuner“. Der vor allem vom Zentralrat deutscher Sinti und Roma in Heidelberg angemahnte konsequente Gebrauch des Terminus „Roma“ ist jedoch nicht unproblematisch, da dieser im Romanes, der Sprache der Roma/Zigeuner, nur soviel wie „Mensch“ meint. Andere Gruppierungen wie die Lalleri oder die Litautikker lehnen die Bezeichnung „Roma“ für sich ab, haben aber keine Einwände, mit dem von anderen als diskriminierend empfundenen Label „Zigeuner“ bedacht zu werden. Dass der Terminus in der überwiegenden Zahl der Fälle diskriminierend gemeint und auch so empfunden wird, dürfte indes unstrittig sein. Meine Schreibweise reproduziert in dezidiert kritischer Absicht den Gebrauch des Terminus nach den literarischen Vorgaben. Vgl. zur Diskussion der Terminologie Hans Richard Brittnacher, Leben auf der Grenze. Klischee und Faszination des Zigeunerbildes in Literatur und Kunst, Göttingen, Wallstein, 2012.

27. Der Glöckner, S. 70/ NDdP 62: „Si cette jeune fille était un être humain, ou une fée, ou un ange, c'est ce que Gringoire [...] ne put décider dans le premier moment, tant il fut fasciné par cette éblouissante vision."

28. Der Glöckner, S. 348/ NDdP 334: „Trois cœurs d'homme faits différemment“. 
am Ende des Romans nach dem Vorbild von Cervantes' La Gitanilla eben nicht als Zigeunerin erweist, sondern als ein geraubtes Kind aus einem norditalienischen Adelsgeschlecht, das unter Fahrenden aufwuchs und deren Sitten und Bräuche übernahm, so erweist sich auch Esmeralda zuletzt als Scheinzigeunerin, als das der armen Chantefleurie von Zigeunern geraubte Kind.

Die deutschen Übersetzungen von Hugos Roman haben immer - aus naheliegenden Gründen - nicht die vom französischen Titel des Romans bewusst symbolisch und poetologisch bedeutsam codierte Kathedrale NotreDame ins Zentrum gesetzt, ${ }^{29}$ und auch nicht das Schicksal Esmeraldas betont, sondern durchweg den Glöckner als zentralen Protagonisten der Handlung namhaft gemacht: Der Glöckner von Notre-Dame. Tatsächlich ist seine enorme Körperlichkeit, das Nebeneinander von überwältigend starker Physis und erbärmlicher sozialer Geltung ein ästhetisch ungemein reizvoller und sozialgeschichtlich aufschlussreicher Komplex. Während nahezu alles, was an Mignon fremdartig war, einigen der Leser des Romans sogar als „abstoßend“ 30 erschien, bei Esmeralda in Liebeswürdigkeit und exotische Gefälligkeit transformiert wurde, hat das ganze Kapital des Monströsen jetzt jene Figur allein zu tragen, die der Mutter Esmeraldas, der armen Paquette la Chantefleurie, einem Freudenmädchen, als Ersatz für das geraubte Mädchen untergeschoben wurde. Auch er ist ein Findelkind - aber während Mignon und Esmeralda sog. Scheinzigeunerinnen sind, in der Obhut von Zigeunern aufgewachsene Abkömmlinge sesshafter Familien, ist Quasimodo ein in der Obhut der Kirche aufgezogener, veritabler Zigeuner.

Die Entdeckung des Kuckuckskindes durch die verzweifelt nach ihrer geraubten Tochter suchende Mutter hat der Roman dramatisch beschrieben:

Au lieu de sa gentille petite Agnès, si vermeille et si fraîche, qui était un don du bon Dieu, une façon de petit monstre, hideux, boiteux, borgne, contrefait, se traînait en piaillant sur le carreau. Elle cacha ses yeux avec horreur. [...] On se hâta d'emporter le petit pied-bot. ${ }^{31}$

Enttäuscht müssen die Nachbarn zur Kenntnis nehmen, dass der von der Chantefleurie auf den Stufen der Kathedrale ausgesetzte Wechselbalg weder ersäuft noch verbrannt, sondern zunächst exorziert und dann in kirchliche Obhut genommen wird. Aber auch das klösterliche Leben hat dem - nach dem Tag seines Auffindens und seiner kirchlichen Adoption Quasimodo getauften Ungetüm nicht seine abstoßende Körperlichkeit nehmen können. Im Dachgestühl der mächtigen Kathedrale verrichtet Quasimodo zwischen all den Gryllen, Chimären und Wasserspeiern, ein menschliches Monster inmitten steinerner Gefährten, zur Freude der schaulustigen Menge, sein Handwerk: Er läutet die Glocken, die ihn haben ertauben lassen, springt auf sie, reitet auf ihnen und

29. Vgl. dazu Fritz Peter Kirsch, „Die Struktur von Notre-Dame de Paris im Lichte des Kathedralensymbols“, Zeitschrift für deutsche Sprache und Literatur 78, 1968, S. 10-34.

30. So Schillers Eindruck nach seiner Lektüre der Lehrjahre im Brief an Goethe vom 2. Juli 1796. Goethes Werke, HA, Bd. 7, S. 630.

31. NDdP 214. 
klettert mit bewundernswerter Geschicklichkeit und Körperkraft an der Fassade der Kathedrale herum und auf die Türme hinauf.

„Wir wollen den Versuch nicht machen, dem Leser eine Vorstellung zu geben“, ${ }^{32}$ beginnt der Erzähler, scheinbar überfordert vom zu beschreibenden Ausmaß an Monstrosität, um dann doch zu tun, was er zu unterlassen versprach, nämlich

\begin{abstract}
de donner au lecteur une idée de ce nez tétraèdre, de cette bouche en fer à cheval, de ce petit œil gauche obstrué d'un sourcil roux en broussailles tandis que l'œil droit disparaissait entièrement sous une énorme verrue, de ces dents désordonnées, ébréchées çà et là, comme les créneaux d'une forteresse, de cette lèvre calleuse sur laquelle une de ces dents empiétait comme la défense d'un éléphant, de ce menton fourchu, et surtout de la physionomie répandue sur tout cela, de ce mélange de malice, d'étonnement et de tristesse. [...] [T]oute sa personne était une grimace [...]; entre les deux épaules une bosse énorme [...]; un système de cuisses et de jambes si étrangement fourvoyées qu'elles ne pouvaient se toucher que par les genoux $\left[\ldots . . . .^{33}\right.$
\end{abstract}

Von der Menge, die Quasimodo als Attraktion wahrnimmt, wird er, wenn er mit ihr spricht, d.h. die Glocken läutet, durchaus gefeiert und sogar am 6. Januar 1482, als mit dem Dreikönigstag auch das Narrenfest begangen wird, zum Narrenpapst gekrönt - ein furioses Spektakel der karnevalesken Volkskultur, in dem das verhöhnte Monster stoisch den Spott und die Huldigungen der Volksmenge entgegennimmt. Hugos Roman schildert hingerissen und zugleich mit ethnologischer Gründlichkeit das Leben der Armen, der Bettler und der Fahrenden. Die polymorphe Volksmenge ist gleichsam das soziale Äquivalent zu der disparaten Architektur der Stadt und ihrer Kathedrale. ${ }^{34}$ In deutlich poetologischer Absicht erscheint dabei das Leben der deklassierten Städter als ein Karneval der Unterprivilegierten - mit all seinen Charakteristika der sozialen Umcodierung, der derben Komik, der Unzerstörbarkeit des Lebens, dem Ausstellen der Körpers und seiner Extremitäten, wie sie Bachtin als Charakteristika der Volkskultur identifiziert hat. ${ }^{35}$

Solange Quasimodo inmitten dieses Milieus das bleibt, wozu die Natur ihn bestimmt hat, eine bizarre Laune der Natur, deren soziale Anerkennung durch das geduldige Einverständnis mit der eigenen Marginalität erkauft ist, darf er sein beschränktes Leben vergleichsweise unbehelligt führen. Als er jedoch in einem halsbrecherischen Anfall von Wagemut die von der Inquisition als Hexe verfolgte und von dem dämonischen Erzdechanten Frollo verfolgte Esmeralda rettet, wendet sich die Stimmung der Menge gegen ihn. Mit der uneigennützigen Liebe zu Esmeralda - in Wahrheit ist sie die geraubte Agnès -, einer romantischen Variante des alten Märchenstoffs von La Belle et la Bête, nimmt sein Leben

32. Der Glöckner, S. 56.

33. NDdP 51f.

34. Vgl. dazu die aufschlussreichen Beobachtungen von Volker Klotz in dem Kapitel „Stadtschau und Schaustadt" in seiner Monographie: Die erzählte Stadt. Ein Sujet als Herausforderung des Romans von Lesage bis Döblin, München, Hanser, 1969, S. 92-123.

35. Zur Ästhetik von Karneval und Volkskultur vgl. Michail Bachthin: „Der Karneval und die Karnevalisierung der Literatur“, in Bachtin, Literatur und Karneval. Zur Romantheorie und Lachkultur, Frankfurt a. M./ Berlin/ Wien, Ullstein, 1985, S. 47-60. 
als Objekt der Schaulust des Mobs schließlich eine agonale Wendung. Da der Glöckner Esmeralda nicht vor dem Galgen retten kann, folgt er ihr in den Tod. Nach Jahren fand man

\begin{abstract}
[...] deux squelettes dont l'un tenait l'autre singulièrement embrassé. L'un de ces deux squelettes, qui était celui d'une femme, avait encore quelques lambeaux de robe d'une étoffe qui avait été blanche [...]. L'autre, qui tenait celui-ci étroitement embrassé, était un squelette d'homme. On remarqua qu'il avait la colonne vertébrale déviée, la tête dans les omoplates, et une jambe plus courte que l'autre. ${ }^{36}$
\end{abstract}

Ironisch hat Hugo die liebevolle Betreuung der Zigeunerin durch den verkrüppelten Glöckner als „Quasimodos Heirat“ ${ }^{37}$ bezeichnet. Sein Versuch, sich zumindest im Tod das Menschenrecht auf Zuneigung zu erwerben, ist gescheitert. Während der im Roman leitmotivisch eingesetzte Begriff der „Ananké“, den der Erzähler zu Beginn des Romans im Gestein der Kathedrale eingraviert findet - „mit der Hand auf die Wand eingegraben“" ${ }^{38}$-, die Idee eines unausweichlichen Schicksals, der Verzweiflung des dämonischen und unglücklich verliebten Frollo als Chiffre dienen und ihr sogar mythologisches Gewicht geben darf, ${ }^{39}$ muss der Versuch des Monsters, sich seinem Schicksal zu entziehen, in vollständiger Annihilation enden. Was nicht sein soll, muss ohne jeden Rückstand aus der Schöpfung verschwinden: „Als man ihn von dem Skelett, das er umfaßt hielt, losmachen wollte, zerfiel er in Staub.“" ${ }^{40}$ Mignon, immerhin, bleibt im Saal der Vergangenheit als Reliquie einer überwundenen Welt präsent, Quasimodo wird aus der Erinnerung exorziert.

\title{
1869: Gwynplaine (Victor Hugo, L'Homme qui rit)
}

Das Thema körperlicher Abweichung und ihrer sozialen Wahrnehmung hat Victor Hugo auch noch in seinem späten, im Exil entstandenen Roman L'Homme qui rit beschäftigt. Wie Goethes Lehrjahre erlauben auch die beiden Romane Victor Hugos, Notre-Dame so gut wie L'Homme qui rit, die Rekonstruktion spezifischer Einstellungen der sich formierenden bürgerlichen Gesellschaft zu monströser Körperlichkeit, die zwischen Irritation, Faszination und Emphase oszillieren.

Das Schicksal Mignons und Esmeraldas, geraubt und von Schaustellern abgerichtet zu werden, teilt auch Gwynplaine, der Held von L'Homme qui rit (1869), den mit Quasimodo zudem noch das Schicksal der Verstoßung verbindet. Erst spät - auch dies wie bei Mignon und Quasimodo - enthüllt sich bei Gwynplaine das Geheimnis seiner (aristokratischen) Herkunft. Sehr viel drastischer noch war indes sein Schicksal: Comprachicos - so nennt Hugo eine geheimnisumwitterte

\footnotetext{
36. NDdP 499-500.

37. Der Glöckner, S. 532/ NDdP 498: „Mariage de Quasimodo“.

38. Der Glöckner, S. 5/ NDdP 3: „ce mot gravé à la main sur le mur“.

39. Zum leitmotivischen Charakter der Ananke vgl. Richard B. Grant, The Perilous Quest. Image, Myth and Prophecy in the Narratives of Victor Hugo, Durham N.C., Duke University Press, 1968.

40. Der Glöckner, S. 534/ NDdP 500: „Quand on voulut le détacher du squelette qu'il embrassait, il tomba en poussière."
} 
Bande von professionellen Kinderdieben, die bei dynastischen Händeln aktiv werden, um unerwünschte Erben aus dem Weg zu schaffen ${ }^{41}$ - haben den Jungen nicht nur geraubt, sondern nach einer in arkanen Schriften überlieferten Anweisung das Gesicht zu einer Fratze des immerwährenden Lachens entstellt: „Bussa fissa usque ad aures, genzivis denudatis, nasoque murdridarto, masca eris, et ridebis semper.“ ${ }^{22}$ Eine Fußnote teilt die Übersetzung mit: „Die Backe wird gespalten bis zu den Ohren, das Zahnfleisch entblößt und die Nase abgeschnitten; dann wirst Du eine Maske sein und immerfort lachen." 43

Die Praktiken der Comprachicos waren jenen, die Alexandre Dumas in L'Homme au masque de fer - zehn Jahre vor Hugo - beschrieben hat, ${ }^{44}$ in dem ein Zwillingsbruder des Sonnenkönigs im Kerker hinter einer eisernen Maske schmachtet, weit überlegen: „Die eiserne Maske läßt sich herunterreißen, die fleischerne nicht." 45

Eine Wendung der politischen Ereignisse jedoch, damit lässt Hugo seinen Roman beginnen, hat die Comprachicos gezwungen, aus dem Land zu fliehen. Um sich aller belastenden Indizien zu entledigen, überlassen sie in einer stürmischen Winternacht ein verunstaltetes Kind seinem Schicksal und segeln davon. Gwynplaine, dessen Gesicht nach der oben beschriebenen Anweisung zu einer lachenden Maske entstellt ist, überlebt, findet im Schneesturm die Leiche einer erfrorenen Bettlerin, die kurz zuvor entbunden hat, und nimmt sich liebevoll des neugeborenen Mädchens an. Ihren Frieden finden die Kinder bei dem Gaukler Ursus und seinem Wolf Homo: Der Gaukler trägt den Namen eines Tieres, des Bären, sein Wolf den des Menschen: Homo. Die Sprache des Romans macht die Protagonisten zu Monstren, „zum Tiermenschen und zum vermenschlichten Tier“, ${ }^{46}$ seine Handlung freilich zeigt, dass monströs nur die Gebräuche der Sesshaften und Wohlhabenden sind, nicht die der Fahrenden, die einander in brüderlicher Kreatürlichkeit beistehen. Denn Ursus zieht die beiden Kinder auf und ermöglicht ihnen eine bescheidene Karriere in der Welt der Jahrmärkte. Das

41. Das Delikt - die Verstümmelung ,jussu regis“, auf Befehl des Königs - hat es durchaus gegeben, aber der professionelle Zusammenschluss der Täter zu einer international operierenden Bruderschaft von Kinderdieben dürfte wohl eine Erfındung Hugos sein. Vgl. Rainer G. Schmidt, „Ges[ch]ichtsklitterung. Stichpunkte zu Der Mann mit dem Lachen (= Nachwort)“, in Victor Hugo, Der Mann mit dem Lachen, aus dem Franz. von Rainer G. Schmidt, Bd. 2, Butjadingen/ Brüssel/ Hamburg, Achilla-Presse, o. J., S. 407-422, hier S. 412; vgl. auch Thomas Macho, „Zoologiken: Tierpark, Zirkus und Freakshow“, in Gert Theile (Hrsg.), Anthropometrie, München, Wilhelm Fink Verlag, 2005, S. 155-179, hier S. 175.

42. Victor Hugo, L'Homme qui rit, hrsg. von Marc Eigeldinger und Gérald Schaeffer, 2 Bde, Paris, Flammarion, 1982. Nach dieser Ausgabe zitiere ich mit der Sigle Lqr, der Bandangabe in römischen und der Seitenzahl in arabischen Ziffern. Hier Lqr I, 240.

43. Ich zitiere nach der deutschen Ausgabe: Victor Hugo, Die lachende Maske, Berlin, Verlag Schwarzer Nachtschatten, o.J., S. 114. Lqr I, 419: „La bouche fendue jusqu'aux oreilles, les gencives mises à nu, le nez écrasé, tu seras un masque, et tu riras toujours.“

44. Alexandre Dumas (père), Le Vicomte de Bragelonne ou L'Homme au masque de fer (1845/46).

45. Die lachende Maske, S. 21/ Lqr I, 75: „le masque de fer est arrachable, le masque de chair ne l'est pas."

46. Andreas Fliedner, „Nachwort“, in Victor Hugo, Der lachende Mann, deutsch von Georg Büchmann, Bd. 4, Berlin, Golkonda, 2013, S. 213-231, hier S. 218. 
Mädchen, das bei den dramatischen Umständen seiner Geburt erblindet ist, wird Dea genannt und wächst zu einer Schönheit heran. Sie, die Blinde, sieht nur die Schönheit von Gwynplaines Seele und verliebt sich in den Gaukler, den seine Grimasse trotz des erlittenen Schicksals und selbst im Angesicht größten Leids zum Lachen verurteilt: ${ }^{47}$

Gwynplaine était saltimbanque. Il se faisait voir en public. Pas d'effet comparable au sien. Il guérissait les hypocondries rien qu'en se montrant. Il était à éviter pour des gens en deuil, confus et forcés, s'ils l'apercevaient, de rire indécemment. Un jour le bourreau vint, et Gwynplaine le fit rire. On voyait Gwynplaine, on se tenait les côtes ; il parlait, on se roulait à terre. Il était le pôle opposé du chagrin. Spleen était à un bout, et Gwynplaine à l'autre. ${ }^{48}$

Die Eintracht der so gegensätzlichen Akteure, der zauberhaften Blinden und des verstümmelten Mannes, macht Gwynplaine und Dea zur artistischen Sensation: „Er war das Grauen, sie war die Anmut.“ ${ }^{49}$ Die Verbindung des Schreckens und der Schönheit illustriert - wie in Notre-Dame das Nebeneinander von Esmeralda und Quasimodo - jene Harmonie der Gegensätze, die Hugo im Préface zu Cromwell (1827) als Ausweis wahrer romantischer Imagination gefordert hatte. Aber Hugo rechtfertigt nicht allein eine groteske Ästhetik, die ihren Erfolg dem Reiz des Gegensätzlichen verdankt, sondern bewahrt seiner Prosa trotz aller romantischen Imagination die realistische Nähe zu der an Schönheit und Glück

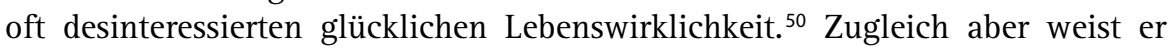
mit diesem „Liebespaar der komplementären Körperbehinderungen“"51 bereits weit voraus in ein Zeitalter, das die Ästhetik eines beschädigten Lebens erst zu entdecken beginnt. ${ }^{52}$

Zusammen mit Ursus, einem philosophisch gebildeten Gaukler und Überlebensvirtuosen, dem treuen Wolf Homo und zwei Landstreicherinnen leben Dea und Gwynplaine das Leben der Fahrenden - in der Parallelwelt des Jahrmarkts genießen sie Bürgerrecht. Während Goethe in den Lehrjahren die Brutalität im Leben der Fahrenden hervorhebt, die Mignon misshandeln, während Hugo in Notre-Dame das zugleich gutmütige und brutale karnevalistische Treiben des Volkslebens und seiner Parallelgesellschaft der Asozialen beschwört, idealisiert Hugo in L'Homme qui rit die counterculture der Fahrenden als Utopie. Als Monster meidet Gwynplaine die Öffentlichkeit und streift nur des nachts durch

47. Vgl. zum vieldeutigen Archetypus der unfreiwilligen Komik (des Pierrot, der Maske, des Gewaltclowns etc.) die umfassende Monographie zur Ästhetik des Zirkus von Anna-Sophie Jürgens, Poetik des Zirkus. Die Ästhetik des Hyperbolischen im Roman, Heidelberg, Carl Winter, 2016 , v. a. S. $228 \mathrm{ff}$.

48. Lqr I, 340.

49. Die lachende Maske, S. 183/ Lqr I, 351: „Il était l'horreur, elle était la grâce.“

50. Vgl. dazu William Wolfgang Holdheim, Die Suche nach dem Epos. Der Geschichtsroman bei Hugo, Tolstoi und Flaubert, Heidelberg, Carl Winter, 1978, S. $16 f$.

51. Tobias 0. Meißner, „Unregelmäßigkeit statt Ebenmaß. Ein Vorwort“, in Hugo, Der lachende Mann, S. 7-13, hier S. 9.

52. Man denke etwa an den Erfolg der paralympics. Vgl. dazu Lorenz Jäger, Beschädigte Schönheit. Eine Ästhetik des Handicaps, Springe, Zu Klampen, 2014, v.a. S. 55-59. 
die verlassenen Straßen der Stadt, unter einem Hut mit tief herabgeschlagener Krämpe - unbedeckt lässt er sein Gesicht nur auf dem Theater sehen.

Nur inmitten anderer „displaced persons“ 53 können Dea und Gwynplaine ungefährdet leben - und in der Greenbox, ihrem Theater, zur Gaudi des Publikums ein Spektakel inszenieren, das ihre eigene Geschichte nachstellt: Die im Wald verirrte Unschuld begegnet einem Ungeheuer - ein Stoff, der nicht zufällig die Geschichte von Esmeralda und Quasimodo wiederholt, aber auch überbietet und wie dort schon an das Märchen von La Belle et la Bête erinnert und damit eine märchenhafte, unvordenkliche Eintracht aller Geschöpfe beschwört. Dea, das verlassene und in der Kälte zitternde Kind stößt in finsterer Nacht auf ihren verunstalteten Helfer und findet damit Trost in ihrer Einsamkeit:

Tout son cœur se fondait en un ineffable amour. Elle se sentait hors de danger, elle trouvait le sauveur. Le public croyait voir le contraire. Pour les spectateurs, l'être sauvé, c'était Gwynplaine, et l'être sauveur, c'était Dea. [...] Et Dea [...], adorait l'ange, pendant que le peuple contemplait le monstre [...]. ${ }^{54}$

Der Kontrast von Grauen und Schönheit und die Aufrichtigkeit der Liebe beider begründet den immensen Erfolg ihres Auftritts: „Man fühlte, dass sie ihr Ungetüm liebte.“ ${ }^{55}$

Während die rohe Volksseele das Glück in der grotesken Idylle begreift, sieht die Herablassung der besseren Kreise nur den so vergeblichen wie erheiternden Versuch des Hässlichen, am Schönen teilzuhaben. Während Goethe in den Lehrjahren deutlich für die kultivierte Geselligkeit und moderate Humanität einer gehobenen Kultur plädiert, die sich voller Mitgefühl, wenn auch vergeblich der devianten Figuren des Romans (neben Mignon ist dies auch der Harfner) annimmt, während Hugo in Notre-Dame das Volkstreiben als karnevalesken Ort sozialer Turbulenz feiert, bei dem ihn eher die „rotwelsche Hierarchie“ 56 interessiert, steigert sich seine Gesellschaftskritik in L'Homme qui rit zur drastischen Verwerfung aristokratischer Überheblichkeit. Die kultivierte Intelligenz bei Goethe betrachtet das körperlich Abnorme als Gegenstand ästhetischen Interesses und wissenschaftlicher Neugierde, der Pöbel in Victor Hugos Notre-Dame lebt mit dem Monster, lacht mit ihm, und quält es auch gelegentlich. Die Aristokratie in L'Homme qui rit hingegen amüsiert sich grundsätzlich und immer über den Abweichenden. Nichts charakterisiert in Hugos Augen mehr den verdorbenen Charakter der englischen Aristokratie als ihre eigentümlichen Interessen: „In den Sitten jener Zeit spielte die Vorliebe für das Mißgestaltete eine Rolle, besonders bei den Frauen“. ${ }^{57}$ Die englische Oberschicht „hatte eine leidenschaftliche Vorliebe für alle Vorführungen auf Straßen und Plätzen, für Gauklerbühnen und Buden mit seltsamen Tieren, für Seiltänzer, Spaßmacher, Hanswürste und

53. Fliedner, „Nachwort“, S. 221.

54. Lqr I, 380.

55. Die lachende Maske, S. 201/ Lqr I, 379: „On sentait qu'elle aimait son monstre.“

56. Klotz, Erzählte Stadt, S. 115.

57. Die lachende Maske, S. 135/ Lqr I, 268: „Dans ces mœurs-là, le goût du difforme existait, particulièrement chez les femmes [...].“ 
Narren, für Possenspiele und Jahrmarktswunder“ ${ }^{58}$ Die Aristokratie nimmt bei ihren Ausflügen in die Hefe des Volkes nicht etwa Anteil am Schicksal der Unterprivilegierten, sie amüsiert sich vielmehr über deren vergebliches Streben nach Normalität.

Alles in Hugos Roman ist monströs, nur nicht die vermeintlichen Monster: In Wahrheit sind sie die schönen Seelen, während die bizarren Rituale der parlamentarischen Routine genauso wie der Spleen der happy few, sich auf Kosten der Elenden zu amüsieren, das Abbild wahrer Monstrosität liefern, die „in den entstellten Zügen des lachenden Mannes seine exemplarische Gestalt gewinnt.“ ${ }^{59}$ Von Gwynplaine zum Joker, dem Gegenspieler Batmans in den Comics von Bobe Cane und Bill Finger, ist es nur ein kleiner Schritt: ${ }^{60} \mathrm{Er}$ wird die Richtung des Lachens umkehren. Im Joker lacht der Clown über eine Gesellschaft, die sich bislang auf seine Kosten amüsierte.

In Goethes Lehrjahren enthüllt am Ende des Romans die nachgetragene Vorgeschichte die Herkunft Mignons als eines im Inzest gezeugten Adelskindes. Auch in L'Homme qui rit ist es eine Analepse, die zur Aufklärung der Vorgeschichte Gwynplaines führt, allerdings auch zum tragischen Ausgang des Geschehens. Der Fund einer an Land gespülten Flaschenpost, in der die Comprachicos einst, den Untergang im Sturm vor Augen, ihre Verbrechen gestanden und niedergeschrieben haben, ermöglicht die Rehabilitation Gwynplaines als Aristokrat. „Kurz, der gelehrte Kanzler beantragte die Wiedereinsetzung in alle Güter und Würden des Lord Fermain Clancharlie, fälschlich Gwynplaine genannt, unter der einzigen Bedingung, daß er dem Missetäter Hardquanonne gegenübergestellt und von besagtem Hardquanonne wiedererkannt würde." ${ }^{61}$ Doch die Nobilitierung reißt Gwynplaine aus dem einzigen Milieu, in dem zu leben ihm möglich war. Die gnadenlose Welt des englischen Hofes kann ihn nur als Monster sehen, macht sich seinen Anblick als satanische Reverenz - als „Abbild des großen Höllenlachens“62 - oder als mythologische Anspielung - als „Angesicht des Prometheus, entstellt durch die Schnabelhiebe des Geiers“63 erträglich. Dass einer der ihren, aus ihren Kreisen verstoßen, ihnen nun mit seiner aus dem eigenen Fleisch geschnittenen Maske die eigene moralische Entstellung vor Augen führt, ist ein Skandalon, das nach erneuter Verstoßung verlangt.

58. Die lachende Maske, S. 143/ Lqr I, 281: „aimait passionnément les exhibitions de carrefours, les tréteaux à parade, les circus à bêtes curieuses, les baraques de saltimbanques, les clowns, les tartailles, les pasquins, les farces en plein vent et les prodiges de la foire."

59. Fliedner, „Nachwort“, S. 219.

60. Es war im übrigen die Darstellung Gwynplaines durch Conradt Veidt in der amerikanischen Verfilmung des Romans The Man Who Laughs (1928), von Paul Leni, die als Vorlage für den Joker bezeugt ist. Vgl. Tobias 0. Meißner, „Vorwort“, S. 8.

61. Die lachende Maske, S. 298/ Lqr II, 128f.: „Bref, le savant chancelier concluait à la réintégration en tous ses biens et dignités de Fermain, lord Clancharlie, faussement appelé Gwynplaine, ,à la seule condition qu'il fût confronté avec le malfaiteur Hardquanonne et reconnu par ledit.“”

62. Die lachende Maske, S. 354/ Lqr II, 217: „la vision du grand rire infernal“.

63. Die lachende Maske, S. 390/ Lqr II, 283: „la face de Prométhée, ravagée par les coups de bec du vautour“. 
Gwynplaines Philippika gegen die Herzlosigkeit der besseren Kreise vor dem englischen Parlament führt angesichts seines Antlitzes, „hinter dem das Grinsen sich bäumte wie ein wildes Pferd, das ausbrechen will“64, zu einem gewaltigen Heiterkeitsausbruch:

On eût pu se croire à la Green-Box. Seulement, à la Green-Box le rire fêtait Gwynplaine, ici il l'exterminait. Tuer, c'est l'effort du ridicule. Le rire des hommes fait quelquefois tout ce qu'il peut pour assassiner. ${ }^{65}$

Gwynplaine kann dem Unrecht, das an ihm begangen wurde, nicht entkommen. Das Lachen, das doch ein Ausdruck kreatürlichen Glücks sein sollte, ihm schon als Kind ins Gesicht geschnitten von seinen Peinigern, verhindert bis zuletzt sein Glück. So gesehen ist Gwynplaine der literarische Archetypus einer modernen Imago: des tragischen Clowns. Äußerlich ist er $\mathrm{zu}$ einem Lachen verurteilt, während seine Seele leidet. Gwynplaine beendet aus freiem Entschluss sein Leben auf dem stürmischen Meer und trotzt mit diesem Akt der Selbstauslöschung jeder erbaulichen Erinnerung.

\section{Was bleibt}

An Mignon und damit an den unversöhnlichen Gegensatz von Poesie und Prosa, von Zauber und Nutzen, erinnert zuletzt noch ein Präparat. In Notre-Dame bleibt vom Monster nur das zu Staub zerfallene Skelett. In L'Homme qui rit hingegen hat Gwynplaine in einem paradoxen Akt der Selbstauslöschung sich selbst jeder erbaulichen Erinnerung widersetzt: Das Monster ist Subjekt geworden, um sich selbstbestimmt der Hypocrisie des Gedenkens zu entziehen. Nur die Literatur hält den Schatten fest, den es geworfen hat und erinnert an seine Anklage einer mörderischen sozialen Rücksichtslosigkeit, die den, der anders ist, allenfalls in Parallelgesellschaften - auf dem Jahrmarkt, dem Zirkus, der Freakshow in Frieden lässt.

Goethes Roman beschreibt nicht nur, wie das Fremde und Abweichende pathologisiert und regelrecht zu Tode therapiert wird, er tötet es ein zweites Mal, indem er es im ästhetischen und akademischen Gedenken als bemerkenswerte Anomalie überdauern lässt. Hugos Romane holen das eskamotierte Monströse in die Lebenswirklichkeit, die seine Romane abbilden, zurück, indem sie Partei für das Monstrum ergreifen, ohne seine Anstößigkeit zu verschweigen, die immer wieder eliminatorische Energien aktiviert.

Diese unterschiedliche Sicht auf das Monströse hat Konsequenzen für den Stil der Prosa: Die Diktion Goethes bleibt, auch dort, wo sie das Schicksal Mignons bedauert, kühl und dem Geist der Aufklärung verhaftet und nimmt in ratloser Resignation die enigmatische Andersheit Mignons hin. Hugos Prosa

64. Die lachende Maske, S. 391/ Lqr II, 284: „sous laquelle se cabrait le rictus, comme un cheval sauvage prêt à s'échapper.“

65. Lqr II, 290. 
hingegen erlaubt keine Distanz: Sie geht mit ihren Figuren gleichsam durch dick und dünn und demonstriert dabei romantisches Pathos, aber auch realistische Abgeklärtheit. Goethes Schauplätze sind - mit Ausnahme des zweiten Buches, das sich der Fülle und den Farben des Lebens aussetzt ${ }^{-66}$ solche einer räsonierenden Öffentlichkeit, das Theater der sich formierenden bürgerlichen Welt oder die Salons und Teestuben aristokratischer Lustschlösser, wo im gepflegten Gespräch die Ansprüche des Lebens im Für und Wider kultivierter Geselligkeit leidenschaftslos und diszipliniert erörtert werden. Hugo hingegen taucht ein in die Turbulenz des städtischen Erlebnisraums, auch seiner Keller, seiner Marktplätze und Jahrmärkte, aber auch in die sogenannten besseren Kreise und verhilft dem Argot der Volksmenge, dem Idiolekt der Asozialen und Fahrenden, aber auch dem zynischen Diskurs der upper class zum Ausdruck, aber perspektiviert ihn mit der ausdauernden Geduld des realistischen Erzählens.

Die Literaturgeschichte des Monstrums kennt viele Beispiele, aber weiß sich keinen Rat. Hugos Romane, das unterscheidet sie von Goethe, der sich mit der Unbegreiflichkeit des Monströsen abfindet, gibt den Monstern eine Stimme und beklagt ihr Schicksal als das des Besonderen in einer zunehmend mediokren Welt.

66. Max Kommerells Interpretation hat sich ganz auf das Zweite Buch der Lehrjahre beschränkt und damit die Interpretation von Karl Schlechta, die noch heute als einer der wichtigsten Erträge der Forschung gelten darf, vorbereitet: Max Kommerell, „Wilhelm Meister“, in Kommerell, Essays, Notizen, poetische Fragmente, hrsg. von Inge Jens, Olten/ Freiburg, Walter, 1969, S. 81-186. Karl Schlechta, Goethes Wilhelm Meister, Frankfurt a. M., Vittorio Klostermann, 1953. 